Mon. Not. R. Astron. Soc. 000,1 19 (2014) Printed 28 September $2018 \quad$ (MN LTEX style file v2.2)

\title{
A new double radio relic in PSZ1 G096.89+24.17 and a radio relic mass-luminosity relation
}

\author{
F. de Gasperin ${ }^{1}$, R. J. van Weeren ${ }^{2}$, M. Brüggen ${ }^{1}$, F. Vazza $^{1}$, A. Bonafede ${ }^{1}$, H.T. Intema ${ }^{3}$ \\ ${ }^{1}$ Universität Hamburg, Hamburger Sternwarte, Gojenbergsweg 112, D-21029, Hamburg, Germany \\ ${ }^{2}$ Harvard-Smithsonian Center for Astrophysics, 60 Garden Street, Cambridge, MA 02138, USA \\ ${ }^{3}$ National Radio Astronomy Observatory, 1003 Lopezville Road, Socorro, NM 87801-0387, USA
}

\begin{abstract}
Radio relics are diffuse synchrotron sources in galaxy clusters that are believed to trace large-scale shock waves. We have discovered a new double radio relic system in PSZ1 G096.89+24.17 $(z=0.3)$ and have carried out a full-polarization radio observation using the Westerbork Synthesis Radio Telescope (WSRT) at 1.4 GHz. The observation revealed the presence of two relics located on the two diametrically opposite sides of the cluster and hints of a central radio halo. The linear sizes of the relics are $\sim 0.9$ and $\sim 1.4 \mathrm{Mpc}$.

We carried out an analysis of all known double radio relics by using radio, X-ray and SunyaevZeldovich (SZ) data. We find that the radio luminosity of double relics is a steep function of the cluster mass, with $L_{R} \propto M^{2.83 \pm 0.39}$. If we include single radio relics, this relation is maintained. This dependence has implications for the origin of magnetic fields at the relic's locations.
\end{abstract}

Key words: galaxies: clusters: individual: PSZ1 G096.89+24.17 - large-scale structure of Universe - radio continuum: general

\section{INTRODUCTION}

Giant radio relics are extended sources of radio emission found in galaxy cluster outskirts and are believed to trace shock waves generated during clusters mergers. Shock waves are sites for particle acceleration via the diffusive shock acceleration (DSA) mechanism (Drury 1983, Blandford \& Eichler 1987, Jones \& Ellison 1991). Relics are expected to be preferably found in cluster outskirts because the kinetic energy dissipated in merger shocks peaks at a distance of about half of the virial radius from the cluster centre (Vazza et al. 2012).

Of a particular interest are those systems showing two radio relics, usually found on opposite sides of the cluster. These systems are believed to be the result of a binary merger where the merger axis is nearly perpendicular to the line of sight. Owing to the rather clear geometry of the system, double radio relics can constrain some important quantities as the mass ratio and the impact parameter of the merger (Roettiger, Burns \& Stone 1999, van Weeren et al. 2012b), as well as the efficiency of electron acceleration (Bonafede et al. 2012).

van Weeren et al. (2009b) and Bonafede et al. (2012) studied the correlation of the linear size of radio relics in double systems against the radio power, the projected distance and the spectral index. They found that relics with high radio power have larger linear sizes and that there is a tendency of larger relics to be mostly located at larger distances from the cluster centre and of smaller relics to have steeper spectral indices. These tendencies can be explained by the fact that the larger shock waves occur mainly in lower-density and lower-temperature regions, and have hence larger Mach numbers (i.e. a flatter spectra; Skillman et al. 2008, Vazza, Brunetti \& Gheller 2009). However, the number of double radio relics discovered so far is limited to 15 (Feretti et al. 2012. Bonafede et al. 2012) and the statistical significance of the aforementioned correlations is barely sufficient to put loose constraints on models (Bonafede et al. 2012).

Up to now only around 50 clusters hosting radio relics are known and these are located principally at moderate redshift $(z \lesssim$ 0.3; Feretti et al. 2012). With the advent of the Planck satellite, many new massive (up to $M_{500}=1.6 \times 10^{15} \mathrm{M}_{\odot}$ ) clusters with $z<1$ were discovered (Planck Collaboration et al. 2013). According to simulations, several hundred relics are expected to be discovered by novel radio telescopes (Nuza et al.|2012).

We performed a search for double radio relic systems in all newly discovered Planck clusters and also all the previously known clusters by visual inspecting multiple radio surveys (NVSS ${ }^{1}$ WENSS $S^{2}$ VLSS 3 at the position of know galaxy clusters (1743 objects from the MCXC catalogue and 1227 from the Planck catalogue). Here we report the first outcome of this search which is the discovery of a double radio relic system in the PSZ1 G096.89+24.17 cluster.

In Sec. 2 we present the WSRT observation and data reduction. In Sec. 3 we present the results of these observations. In Sec. 4

\footnotetext{
1 NRAO VLA Sky Survey (Condon et al. 1998)

2 Westerbork Northern Sky Survey, (Rengelink et al. 1997)

3 VLA Low-frequency Sky Survey, (Cohen et al. 2007)
} 
we provide an up-to-date catalogue of all known double radio relics and we discuss the correlations between relics and hosting-clusters properties. Conclusions are in Sec. 5. Throughout this paper we assume a $\Lambda$ cold dark matter $(\Lambda \mathrm{CDM})$ cosmology with $H_{0}=71$ $\mathrm{km} \mathrm{s}^{-1} \mathrm{Mpc}^{-1}, \Omega_{m}=0.27$ and $\Omega_{\Lambda}=0.73$. At the redshift of PSZ1 G096.89+24.17 $(z=0.3) 1^{\prime \prime}$ corresponds to $\sim 4.421 \mathrm{kpc}$. All images are in the $\mathrm{J} 2000$ coordinate system.

\section{OBSERVATIONS}

The cluster was observed with the WSRT on February 7, 2014, for about $12 \mathrm{~h}$ using the default $21 \mathrm{~cm}$ set-up. Due to telescope upgrades, only 8 antennas participated in the observation. A total bandwidth of $160 \mathrm{MHz}$ was recorded, spread over eight spectral windows of $20 \mathrm{MHz}$ in bandwidth. Each spectral window was further subdivided into 64 frequency channels. All four linear polarization products were recorded. The calibrators 3C286 and 3C147 were observed at the start and end of the main observing run, respectively.

The data were calibrated with $\mathrm{CASA}^{4}$ using the flux scale given by Perley \& Butler (2012). The first step consisted of the removal of time ranges affected by shadowing. We then performed an initial bandpass correction using 3C147. Radio frequency interference was removed using the AOFlagger (Offringa, van de Gronde \& Roerdink 2012). This initial bandpass correction prevents flagging data affected by the strong bandpass roll-off. After flagging, we again calibrated the bandpass and subsequently obtained complex gain solutions for the two calibrator sources. The channeldependent polarization leakage terms were calibrated using the unpolarized source $3 \mathrm{C} 137$. The polarization angles were set using 3C286, carrying out a single correction per frequency channel. We then transferred all calibration solutions to the target source. For the target field, three rounds of phase-only self-calibration were performed. The data was imaged taking the spectral index into account during the deconvolution (i.e., nterms $=3$ Rau \& Cornwell 2011). We have made a high-resolution (robust $=0$ ) image and a low-resolution image (robust $=1$ ) to gain sensitivity towards the diffuse emission. The final images were corrected for the primary beam attenuation. In the case of the low-resolution image a dominant source $\sim 30^{\prime}$ to the south caused small artifacts and has been peeled.

The final maps at two different resolutions are shown in Fig. 1 . $15^{\prime \prime} \times 14^{\prime \prime}\left(\mathrm{rms}: 23 \mu \mathrm{Jy}\right.$ beam $^{-1}$, robust=0) and $21^{\prime \prime} \times 20^{\prime \prime}$ (rms: 28 $\mu \mathrm{Jy}_{\text {beam }}{ }^{-1}$, robust $=1$ ).

\section{THE DOUBLE RADIO RELIC IN PSZ1 G096.89+24.17}

PSZ1 G096.89+24.17 (also known as $\mathrm{ZwCl} \mathrm{1856.8+6616)} \mathrm{is} \mathrm{a}$ moderately massive galaxy cluster $\left(M_{500}=4.40_{-0.48}^{+0.45} \times 10^{14} \mathrm{M}_{\odot}\right)$ at $z=0.3$ (spectroscopic measure by Planck Collaboration et al. 2013). The ROSAT unabsorbed X-ray flux is $S_{\mathrm{X}}=1.2 \pm 0.1 \times 10^{-12}$ erg s${ }^{-1} \mathrm{~cm}^{-2}$ in the energy band: $0.1-2.4 \mathrm{keV}$. At the redshift of 0.3 this provides an X-ray luminosity of $3.7 \times 10^{44} \mathrm{erg} \mathrm{s}^{-1}$. Using the $(L, T)$ relations provided by Pratt et al. (2009) we estimated a cluster temperature of $\mathrm{T} \sim 4 \mathrm{keV}$.

The radio emission in the direction of this galaxy cluster is dominated by two strong point sources (one centrally located and one towards north) and by two arc-like extended structures labelled

4 version 4.2, http://casa.nrao.edu

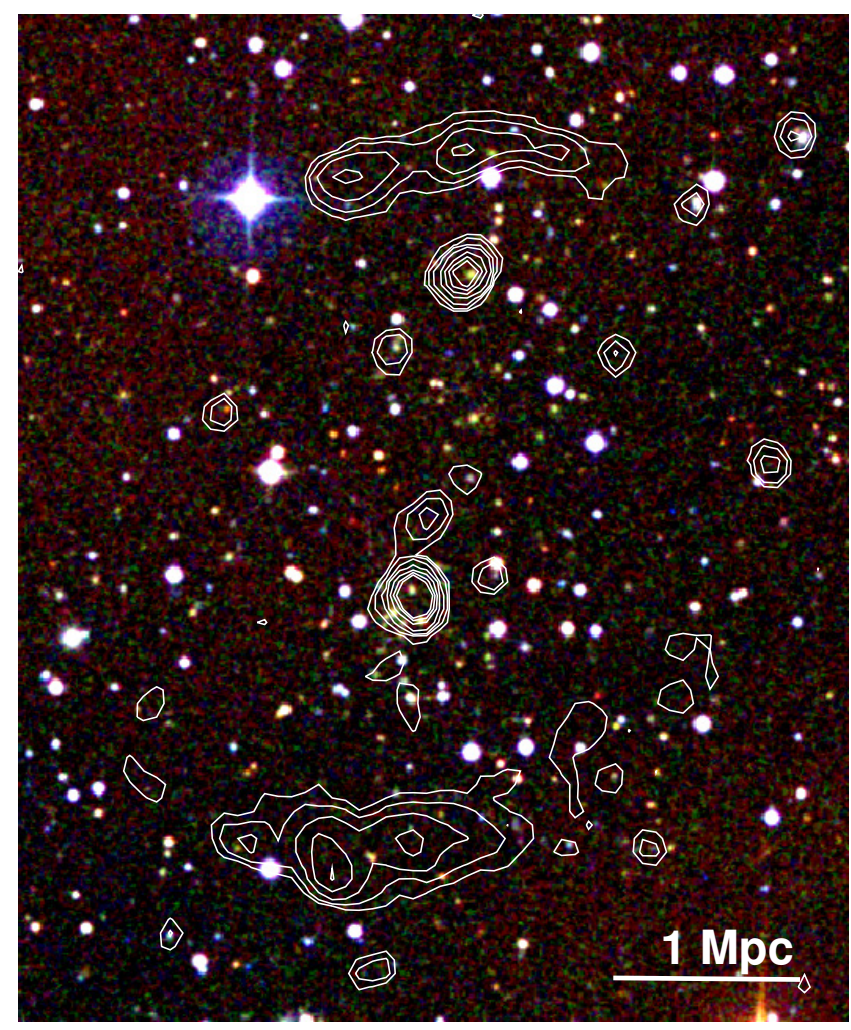

Figure 2. DSS colour image ( $\mathrm{g}, \mathrm{r}$ and $\mathrm{i}$ band) of the cluster with overlaid the WSRT $1.4 \mathrm{GHz}$ contours as in Fig. $1 \mathrm{a}$

Relic $\mathrm{N}$ and Relic S in Fig. 1 Relic $\mathrm{N}$ is about 3.4 long which is around $900 \mathrm{kpc}$ at $\mathrm{z}=0.3$, while relic $\mathrm{S}$ is around 5.5 long which translates into a linear size of $\sim 1.4 \mathrm{Mpc}$. The complete extension of relic $\mathrm{S}$ is visible only in the low-resolution map (Fig. 1b). A composite image from the Digitized Sky Survey (DSS) is shown in Fig. 2 No obvious optical counterpart is visible in the location of the brightest spots of the extended radio emission. The two structures are at a distance of $770 \mathrm{kpc}$ (Relic N) and $1145 \mathrm{kpc}$ (Relic S) from the peak of the X-ray emission (see Fig. 3). The flux of the two extended sources has been extracted from the low-resolution map (Fig. 1b) to ensure minimal flux is lost from the extended emission. Relic $\mathrm{N}$ has a flux density of $8.9 \pm 0.8 \mathrm{mJy}$ while Relic $\mathrm{S}$ has a flux density of $18.3 \pm 1.9 \mathrm{mJy}$. Errors have been calculated by multiplying the map rms by the square root of the number of beams covering the source. We classify the two arc-like sources as radio relics because of (i) their morphology, (ii) their sizes, (iii) the absence of optical counterparts, and (iv) their location and orientation with respect to the cluster centre.

Given the relic average surface brightness, the low signal-tonoise of the $\mathrm{Q}$ and $\mathrm{U}$ Stokes maps would allow a detection only for a fractional polarization $\gtrsim 10 \%$ and only in the brightest spots. A $\sim 10-20 \%$ polarized emission is in fact detected on the brightest spots both in the northern and southern relics (see Fig. 4). Using the map provided by Oppermann et al. (2012) we estimated a Faraday depth in the direction of PSZ1 G096.89+24.17 of 11.6 rad $\mathrm{m}^{-2}$. The polarization angle has therefore been corrected for galactic Faraday rotation by subtracting an angle of $31^{\circ}$. The northern relic appear to have E-vectors mostly aligned perpendicular to the relic extension. This is not the case for Relic $S$ where the vectors are $\sim 45$ apart from being perpendicular to the relic extension.

Assuming that the magnetic field should be aligned with the 


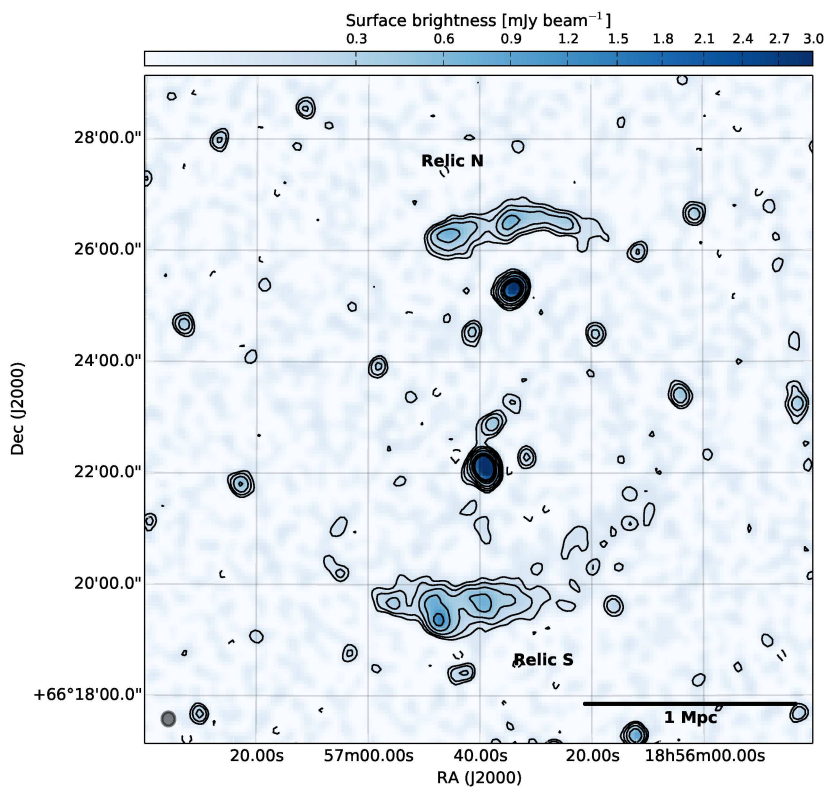

(a) High-resolution $1.4 \mathrm{GHz}$ map

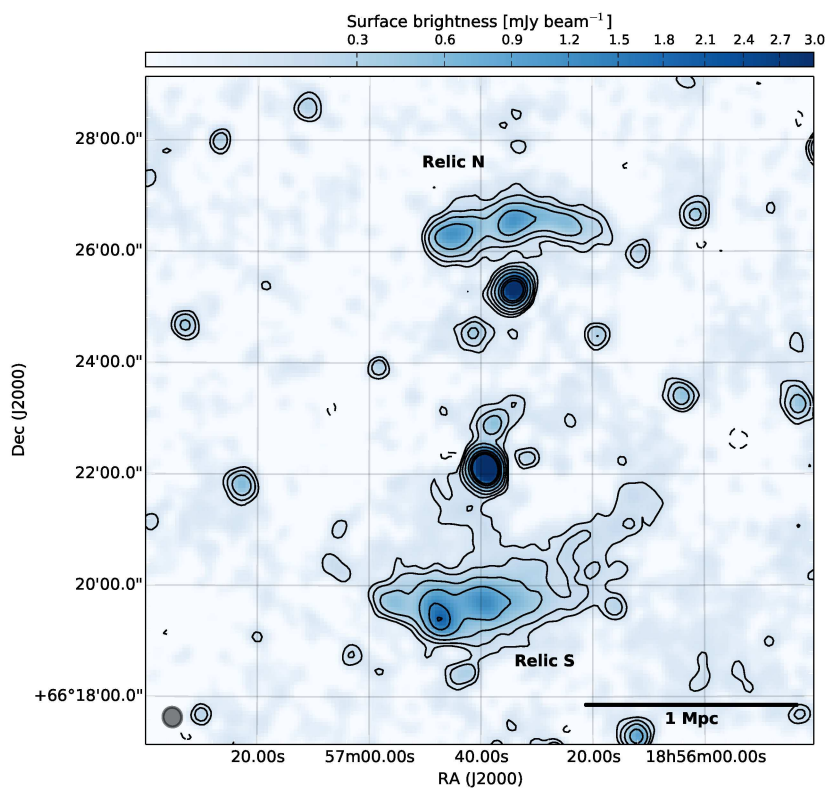

(b) Low-resolution $1.4 \mathrm{GHz}$ map

Figure 1. Left: WSRT $1.4 \mathrm{GHz}$ image. High-resolution (beam: $\left.15^{\prime \prime} \times 14^{\prime \prime}\right)$ map made with robust=0 weighting. Contours are at the level of $(1,2,4, \ldots) \times 3 \sigma_{\mathrm{rms}}$, $\sigma_{\mathrm{rms}}=23 \mu \mathrm{Jy}$ beam $^{-1}$. Dashed contours are at $-3 \sigma$. Right: same map but at lower resolution (robust=1, beam: $21^{\prime \prime} \times 20^{\prime \prime}$ ) to enhance the extended emission. Contours are at the level of $(1,2,4, \ldots) \times 3 \sigma_{\mathrm{rms}}, \sigma_{\mathrm{rms}}=28 \mu \mathrm{Jy}_{\text {beam }}{ }^{-1}$. Dashed contours are at $-3 \sigma$.

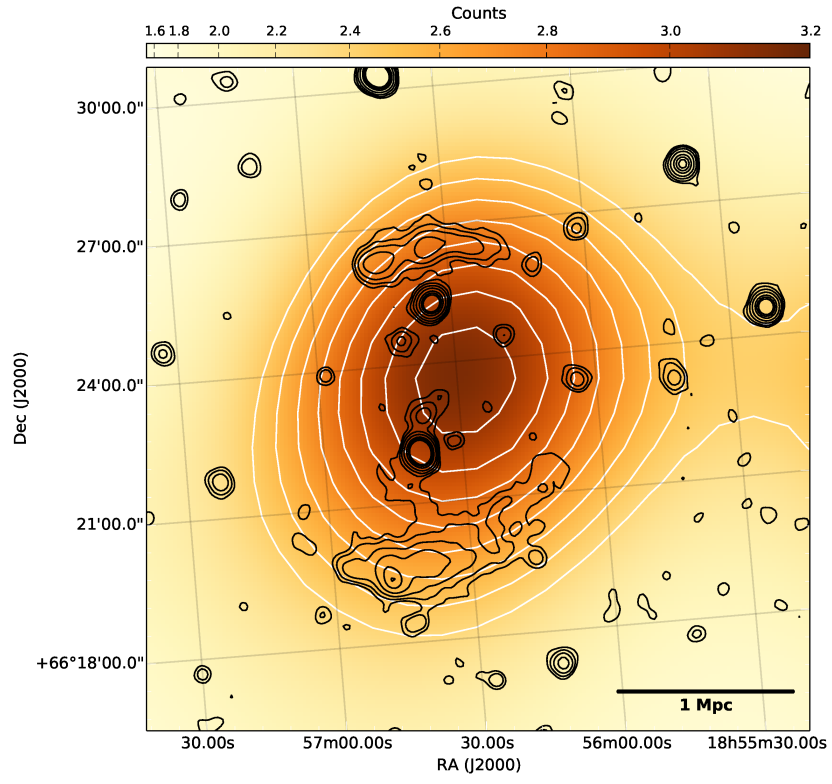

Figure 3. Photon image in the broad band $(0.1-2.4 \mathrm{keV})$ from the ROSAT all sky survey. No other $\mathrm{X}$-ray observations of the cluster are currently available. Image has been smoothed with a two-dimensional Gaussian kernel of $\sigma=4$ pixels $(1$ pixel $=45$ arcsec $)$. White contours are at: $(2.4,2.5, \ldots, 3.3)$ counts. Black contours are from the WSRT $1.4 \mathrm{GHz}$ observation as in Fig. $1 \mathrm{~b}$

relic extension as seen in other radio relics (see e.g., van Weeren et al. 2010), it would produce E-vectors perpendicular to the relic extension, as visible in Relic N. If we consider the merger axis to be slightly tilted, then we expect the ICM to produce a rotation in the polarization angle on the farther relic which should not be present in the closer one. The $45^{\circ}$ misalignment with the polarization vector

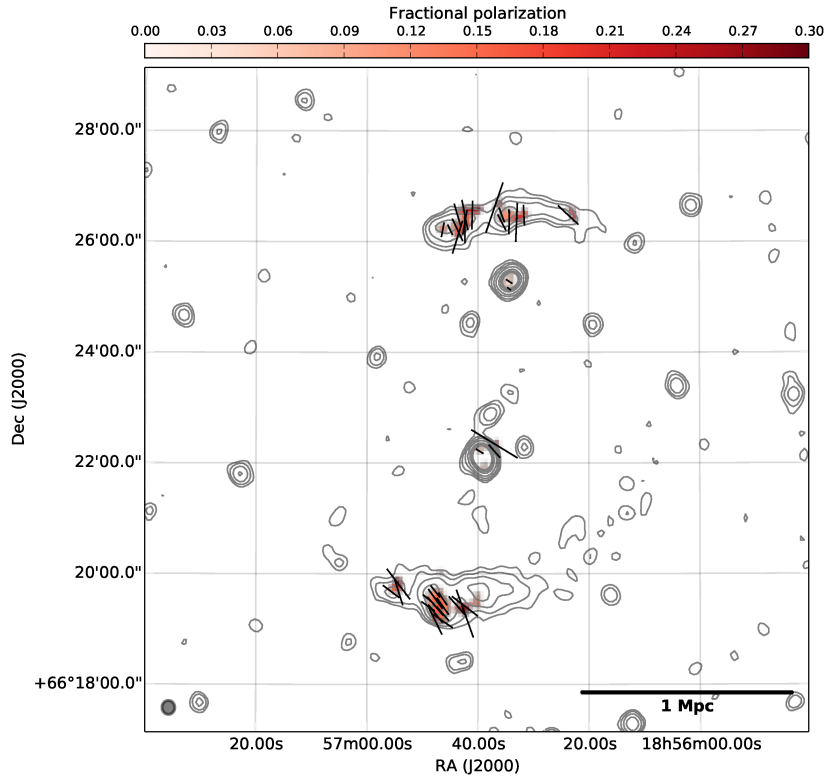

Figure 4. Fractional polarization map with E-vectors displayed. The polarization angle has been corrected for galactic Faraday rotation. Black contours are the WSRT 1.4 GHz as in Fig.1a

in the southern relic compared to the expected direction may be interpreted as a consequence of Faraday rotation. Polarization maps, with rotated polarization vectors in the southern relic compared to the expected orientation, together with the short distance between the relics and the peak of the cluster X-ray emission, suggest that the merge axis is slightly tilted compared to the plane of the sky, with the southern relic farther away from the observer.

Finally, we detect traces of an extended, low-surface brightness feature extending from the two relics towards the centre of the 


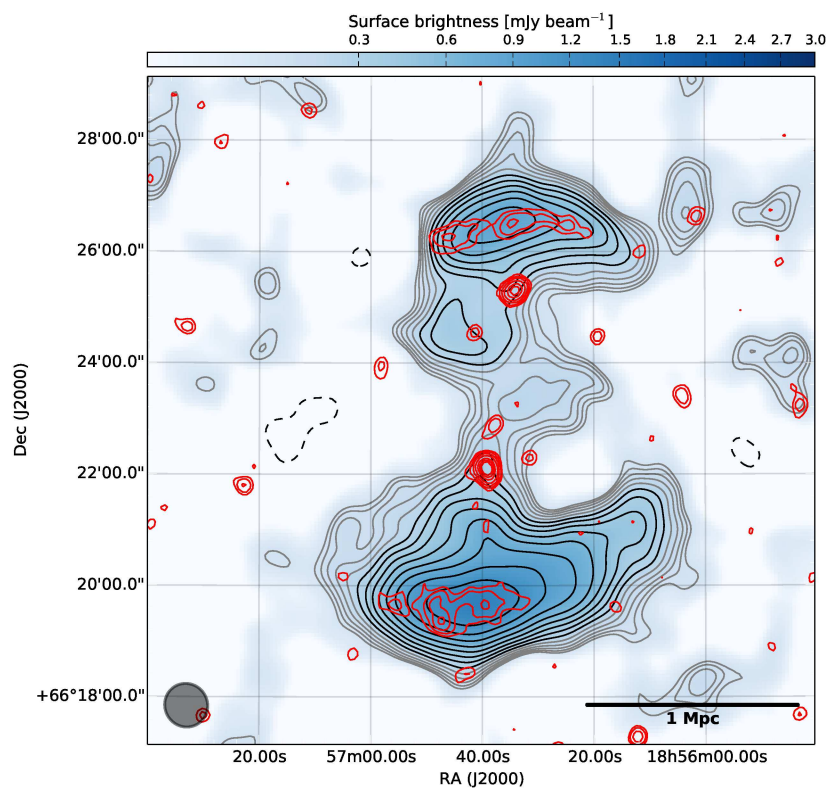

Figure 5. WSRT 1.4 GHz image of the extended radio structure present in PSZ1 G096.89+24.17. The high-resolution image (red contours) has been subtracted and the residuals were imaged tapering the data to a resolution of $46^{\prime \prime}$. Contours are logarithmically spaced between 1 and $20 \sigma$, with $\sigma=$ $90 \mu \mathrm{Jy}$ beam $^{-1}$. Grey contours should be taken with caution since they are below $3 \sigma$. Dashed contours are at $-3 \sigma$.

cluster (see Fig. 5). This can be a hint of a halo which could extend from the cluster centre up to the two radio relics, as in the system MACS J1752.0+4440 (van Weeren et al. 2012a). Planned lowerfrequency observations will confirm its presence. Similar to the case of the bridge in the Coma cluster (Brown \& Rudnick 2011), the radio halo appears to be located in the vicinity of another radio source, implying a possible link.

\section{A CATALOGUE OF DOUBLE RADIO RELICS}

To make a comprehensive study of double radio relic systems, we searched the literature for all double radio relics discovered so far. In table 1 we collected the data of 15 systems, for a total of 30 radio relics. All the lengths and powers have been recalculated using the cosmology of this paper. The luminosities have been k-corrected and scaled to a frequency of $1.4 \mathrm{GHz}$. In cases that no spectral index was available, we assumed a value of $\alpha=-1.3$ Feretti et al. (2012). X-ray luminosities were also scaled to provide powers in the energy range $0.1-2.4 \mathrm{keV}$. We also collected all the available mass estimations obtained by new SZ measurements (Planck Collaboration et al.2013). Abell $1758 \mathrm{~N}$ could host a double radio relic (Giovannini et al.2009) but is not included in our catalogue since higher-resolution images are needed to confirm the presence of the relics.

For Abell 3365, $0217+70$ and CIZA J2242.8+5301 the Planck catalogue does not provide a mass estimate. We used the Malmquist-bias corrected scaling relation provided by Pratt et al. (2009) which gives a mass estimate for a certain X-ray luminosity in the $0.1-2.4 \mathrm{keV}$ range. For the CIZA J2242.8+5301 cluster we obtained $M_{500}=7.97 \pm 0.35 \times 10^{14} \mathrm{M}_{\odot}$ while for Abell 3365 we obtained $M_{500}=2.01 \pm 0.08 \times 10^{14} \mathrm{M}_{\odot}$. Since no radio fluxes are available in the literature for $0217+70$, we exclude this system from the subsequent analysis.

\subsection{Revising old correlations}

van Weeren et al. (2009c) collected the available data in the literature searching for correlations in the radio relics properties. They found that the relic LLS correlates with the projected distance from the cluster centre, with larger relics located at larger distances from the cluster centre, and that the spectral index anti-correlates with the relic LLS, with smaller relics having a steeper spectral indices. These correlations can be explained by the fact that the larger shock waves occur mainly in lower density and lower temperature regions, and hence have larger Mach numbers (Skillman et al. 2008, Vazza, Brunetti \& Gheller 2009).

However, as already recognized by the authors, all length measurements are affected by projection effects. Furthermore, radio phoenices 5 could contaminate the sample adding small-scale, steep spectrum objects that are typically close to the cluster centre.

To overcome these problems, Bonafede et al. (2012) selected only double radio relics, which in the most plausible scenario are consequences of mergers seen in the plane of the sky. This would remove the presence of radio phoenices and minimizes the impact of the projection effect on the measured lengths. They found a good correlation only for the LLS $-L_{1.4 \mathrm{GHz}}$ relation, meaning that relics with high radio power tend to have larger linear sizes.

Here we have repeated their analysis including six new radio relics (three double relic systems), re-measuring all lengths and distances to be consistent with our cosmology and adding the cluster mass taken from the SZ measurements. Linear regression has been made using the BCES bisector method described in Akritas \& Bershady (1996) which accounts for errors in, both, the dependent and independent variable and for the intrinsic scatter of the data.

To access the existence of possible correlations between the selected quantities, we use Spearman's rank correlation coefficient (S-rank). The null hypotheses we are testing is the following: 'there is no association between variable A and variable B'.

The null hypothesis is accepted with a significance level of $83 \%$ for the spectral index - LLS correlation (meaning that no correlation is present) as also found by Bonafede et al. (2012). The same correlation seen in van Weeren et al. (2009c) was most likely driven by the presence of steep-spectrum, centrally located radio phoenices which are excluded from our sample. The null hypothesis is instead rejected (meaning correlation) with a $2 \%$ significance level for the LLS correlation with the cluster distance (see Fig. 6. Although we have recalculated all distances between radio relics and the cluster centre positions assuming the peak of the X-ray emission, this can be misleading since all systems are undergoing a merger which makes it difficult to define a proper cluster centre. Hence, we have recalculated this correlation using the distance between the two radio relics as the independent variable. In this case, we find a better correlation (null hypothesis rejected at $0.03 \%$ significance level, see Fig. 6, which supports the idea that the shock surfaces are larger the further out they are. However, we do not find a significant correlation in the $L_{r}-$ LLS plane as found by Bonafede et al. (2012).

\footnotetext{
5 We consider radio phoenices those emissions generated by fossil radio plasma produced by an old episode of AGN activity. The plasma is compressed, by e.g. a merger shock wave, which boosts both the magnetic field inside the plasma and the momenta of the relativistic particles.
} 
A mass-luminosity relation for radio relics

\begin{tabular}{|c|c|c|c|c|c|c|c|c|c|c|}
\hline Name & $z$ & $\begin{array}{c}\text { Freq } \\
(\mathrm{GHz})\end{array}$ & $\begin{array}{l}\text { Flux } \\
(\mathrm{mJy})\end{array}$ & $-\alpha$ & $\begin{array}{c}L_{1.4 \mathrm{GHz}} \\
\left(10^{23} \mathrm{~W} \mathrm{~Hz}^{-1}\right)\end{array}$ & $\begin{array}{l}\text { LLS } \\
(\mathrm{kpc})\end{array}$ & $\begin{array}{c}d_{c} \\
(\mathrm{kpc})\end{array}$ & $\begin{array}{c}d_{r} \\
(\mathrm{kpc})\end{array}$ & $\begin{array}{c}L_{\mathrm{X}}[0.1-2.4] \\
\left(10^{44} \mathrm{erg} \mathrm{s}^{-1}\right)\end{array}$ & $\begin{array}{c}M_{500} \\
\left(10^{14} \mathrm{M}_{\odot}\right)\end{array}$ \\
\hline A3365 E & 0.0926 & 1.425 & $42.6 \pm 2.6$ & - & 7.4 & 547 & 984 & 1531 & \multirow{2}{*}{0.86} & \multirow{2}{*}{-} \\
\hline A3365 W & 0.0926 & 1.425 & $5.3 \pm 0.5$ & - & 0.9 & 291 & 1021 & 1531 & & \\
\hline ZwCl0008.8+5215 E & 0.1032 & 1.382 & $56 \pm 3.5$ & 1.59 & 15.4 & 1416 & 944 & 1585 & \multirow{2}{*}{0.5} & \multirow{2}{*}{$3.30_{-0.50}^{+0.46}$} \\
\hline $\mathrm{ZwCl} 10008.8+5215 \mathrm{~W}$ & 0.1032 & 1.382 & $11 \pm 1.2$ & 1.49 & 3.0 & 303 & 640 & 1585 & & \\
\hline A2345 E & 0.1765 & 1.425 & $29 \pm 0.4$ & 1.3 & 26.6 & 1565 & 1304 & 2348 & \multirow{2}{*}{4.3} & \multirow{2}{*}{$5.71_{-0.49}^{+0.46}$} \\
\hline $\mathrm{A} 2345 \mathrm{~W}$ & 0.1765 & 1.425 & $30 \pm 0.5$ & 1.5 & 28.5 & 834 & 1096 & 2348 & & \\
\hline A1240 N & 0.1590 & 1.425 & $6 \pm 0.2$ & 1.2 & 4.3 & 866 & 762 & 1802 & \multirow{2}{*}{1.0} & \multirow{2}{*}{$3.71_{-0.54}^{+0.50}$} \\
\hline A1240 S & 0.1590 & 1.425 & $10.1 \pm 0.4$ & 1.3 & 7.3 & 1282 & 1039 & 1802 & & \\
\hline RXCJ1314.4-2515 E & 0.2439 & 0.61 & $28 \pm 1.4$ & 1.41 & 16.8 & 1132 & 1023 & 1425 & \multirow{2}{*}{10.9} & \multirow{2}{*}{$6.15_{-0.73}^{+0.69}$} \\
\hline RXCJ1314.4-2515 W & 0.2439 & 0.61 & $64.8 \pm 3.2$ & 1.40 & 39.0 & 913 & 621 & 1425 & & \\
\hline MACSJ1149.5+2223 E & 0.544 & 1.450 & $4.1 \pm 0.2$ & 1.15 & 53.3 & 775 & 1476 & 2067 & \multirow{2}{*}{14.0} & \multirow{2}{*}{$8.55_{-0.82}^{+0.77}$} \\
\hline MACSJ1149.5+2223 W & 0.544 & 1.450 & $5.6 \pm 0.3$ & 0.75 & 60.3 & 701 & 1108 & 2067 & & \\
\hline MACSJ1752.0+4440 NE & 0.366 & 1.714 & $55.1 \pm 2.9$ & 1.16 & 331 & 1190 & 1091 & 1917 & \multirow{2}{*}{8.2} & \multirow{2}{*}{$6.96_{-0.56}^{+0.53}$} \\
\hline MACSJ1752.0+4440 SW & 0.366 & 1.714 & $25.7 \pm 1.4$ & 1.10 & 150 & 694 & 892 & 1917 & & \\
\hline $0217+70 \mathrm{E}$ & 0.065 & - & - & - & - & 1056 & 1021 & 1972 & \multirow{2}{*}{0.63} & \multirow{2}{*}{ - } \\
\hline $0217+70 \mathrm{~W}$ & 0.065 & - & - & - & - & 1409 & 951 & 1972 & & \\
\hline A3376 E & 0.046 & 1.4 & $122 \pm 10$ & 1.82 & 6.1 & 1180 & 638 & 1824 & \multirow{2}{*}{2.12} & \multirow{2}{*}{$2.27_{-0.21}^{+0.20}$} \\
\hline A3376 W & 0.046 & 1.4 & $113 \pm 10$ & 1.70 & 5.7 & 1039 & 1149 & 1824 & & \\
\hline A3667 E & 0.056 & 1.4 & $300 \pm 20$ & - & 19.6 & 1430 & 1344 & 3347 & \multirow{2}{*}{10.9} & \multirow{2}{*}{$5.77_{-0.24}^{+0.24}$} \\
\hline $\mathrm{A} 3667 \mathrm{~W}$ & 0.056 & 1.4 & $3700 \pm 300$ & 1.1 & 271 & 2000 & 2000 & 3347 & & \\
\hline CIZAJ2242.8+5301 N & 0.192 & 1.4 & $144 \pm 15$ & 1.06 & 150 & 1998 & 1585 & 2576 & \multirow{2}{*}{6.8} & \multirow{2}{*}{ - } \\
\hline CIZAJ2242.8+5301 S & 0.192 & 1.4 & $18 \pm 2$ & 1.29 & 19.5 & 1648 & 1175 & 2576 & & \\
\hline $\mathrm{ZwCl} 2341.1+0000 \mathrm{~N}$ & 0.27 & 0.6 & $14 \pm 3$ & 0.49 & 18.2 & 574 & 738 & 1907 & \multirow{2}{*}{2.4} & \multirow{2}{*}{$5.15_{-0.69}^{+0.64}$} \\
\hline $\mathrm{ZwCl} 2341.1+0000 \mathrm{~S}$ & 0.27 & 0.6 & $37 \pm 13$ & 0.76 & 40.9 & 1230 & 1189 & 1907 & & \\
\hline ACT-CLJ0102-4915 NW & 0.87 & 0.61 & $19 \pm 2$ & 1.19 & 296 & 500 & 1237 & 1590 & \multirow{2}{*}{35} & \multirow{2}{*}{$8.80_{-0.67}^{+0.64}$} \\
\hline ACT-CLJ0102-4915 SE & 0.87 & 0.61 & $3 \pm 0.3$ & 1.4 & 44.8 & 294 & 382 & 1590 & & \\
\hline PSZ1G287.0+32.9 NW & 0.39 & 0.61 & $110 \pm 11$ & 1.16 & 233 & 2482 & 600 & 3135 & \multirow{2}{*}{17.2} & \multirow{2}{*}{$13.89_{-0.54}^{+0.53}$} \\
\hline PSZ1G287.0+32.9 SE & 0.39 & 0.61 & $50 \pm 5$ & 1.33 & 97.1 & 1578 & 2700 & 3135 & & \\
\hline PSZ1G096.89+24.17 N & 0.3 & 1.381 & $8.9 \pm 0.8$ & - & 15.2 & 880 & 770 & 1812 & & \\
\hline PSZ1G096.89+24.17 S & 0.3 & 1.381 & $18.3 \pm 1.9$ & - & 31.2 & 1419 & 1145 & 1812 & 3.7 & $4.40_{-0.48}^{+0.45}$ \\
\hline
\end{tabular}

Table 1. Details of known double radio relics. Col. 1: source name. Col. 2: source redshift. Col. 3: frequency used for the radio flux measure. Col. 4: Radio flux. Col. 5: spectral index (when multiple spectral indices are available, we used the one describing the slope below 1.4 GHz), $S_{v} \propto v^{\alpha}$. Col. 6: k-corrected radio power. Col. 7: Largest linear scale of the relic. Col. 8: distance between the relic and the peak of the X-ray brightness. Col. 9: distance between the two relics. Col. 10: X-ray luminosity. Col. 11: cluster mass measured by the SZ effect.

A3365: van Weeren et al. (2011b); ZwCl0008.8+5215: van Weeren et al. (2011a); A2345: Bonafede et al. (2009); Boschin, Barrena \& Girardi (2010); A1240: Bonafede et al.(2009); RXCJ1314.4-2515: Venturi et al.(2007); MACSJ1149.5+2223: Bonafede et al. (2012); MACSJ1752.0+4440: van Weeren et al. (2012a); Bonafede et al. (2012); 0217+70: Brown, Duesterhoeft \& Rudnick (2011); A3376: Kale et al. (2012); A3667: Rottgering et al. (1997);

Johnston-Hollitt (2004); CIZAJ2242.8+5301 ("Sausage"): van Weeren et al. (2010); Stroe et al. (2013); ZwCl2341.1+0000: Giovannini et al. (2010); van Weeren et al.(2009a); Bagchi et al. (2011b); ACT-CLJ0102-4915 ("El Gordo"): Menanteau et al. (2012); Lindner et al. (2013); PSZ1G287.0+32.9: Bagchi et al. (2011a); Bonafede et al. (2014); PSZ1G096.89+24.17: this work

\subsection{Mass-luminosity correlation}

We have found a remarkably good correlation between the luminosity of the double radio relics and the mass of the host clusters (see Fig. 7). To test this relation further, we have included also single radio relics from Feretti et al. (2012). We excluded those relics classified by the authors as "roundish", which are likely to be radio phoenices, and those relics for whom no Planck SZ mass is given. The error on the radio fluxes for these relics are assumed to be $10 \%$ of the total flux. The correlation in this case is less strong but holds with the null hypothesis rejected at $<0.1 \%$ significance level (see Fig. 7). In both cases, the slope of the regression is $\sim 2.8 \pm 0.4$.

Both samples plotted in Fig. 7 are heterogeneous and affected by several observational and selection biases. All the lowmass $\left(M_{500}<5 \times 10^{14} \mathrm{M}_{\odot}\right)$ clusters are relatively close-by objects $(z \lesssim 0.35)$, although they would be an order of magnitude more abundant in the redshift range $0.35<z<0.9$ than in the local universe due to the larger sampling volume (see e.g., Vikhlinin et al. 2009). This effect is likely driven by a selection bias due to difficulties in finding high-redshift low-mass clusters. On the other other hand, the high-mass end of the sample should have a similar num- ber of high-redshift $(z>0.35)$ and low-redshift $(z<0.35)$ objects, given a lesser impact of the selection bias (Planck Collaboration et al. 2013). We find indeed four double radio relic systems for the high and five for the low redshift bin.

The absence of high-power radio relics in low-mass clusters (top-left corner of Fig. 7) comes from observational evidence: lowmassive clusters are unable to produce high-luminosity radio relics. However, the absence of low-power radio relics in massive clusters (bottom-right corner of Fig. 7) can be an observational bias driven by the distance dependency of the two variables (Malmquist bias). The effect is explained in Fig. 8 where we plot the cluster redshift versus the radio luminosity of radio relics in our sample. The lines show an estimated $1 \sigma$ detection limit for some typical observation set-ups assuming a relic size of $1000 \times 100 \mathrm{kpc}^{2}$ and taking cosmic dimming into account. This plot shows why no lowluminosity relics are detected at high-redshift. However, all massive clusters $\left(M_{500}>5 \times 10^{14} \mathrm{M}_{\odot}\right)$ at moderate redshift lie well above the sensitivity cuts and the known close-by $(z<0.25)$, massive clusters (RXCJ1314.4-2515, CIZAJ2242.8+5301 and Abell 3667) are the only ones at low-redshift that host high-luminosity radio relics. These two facts could give some confidence that the relation is not 


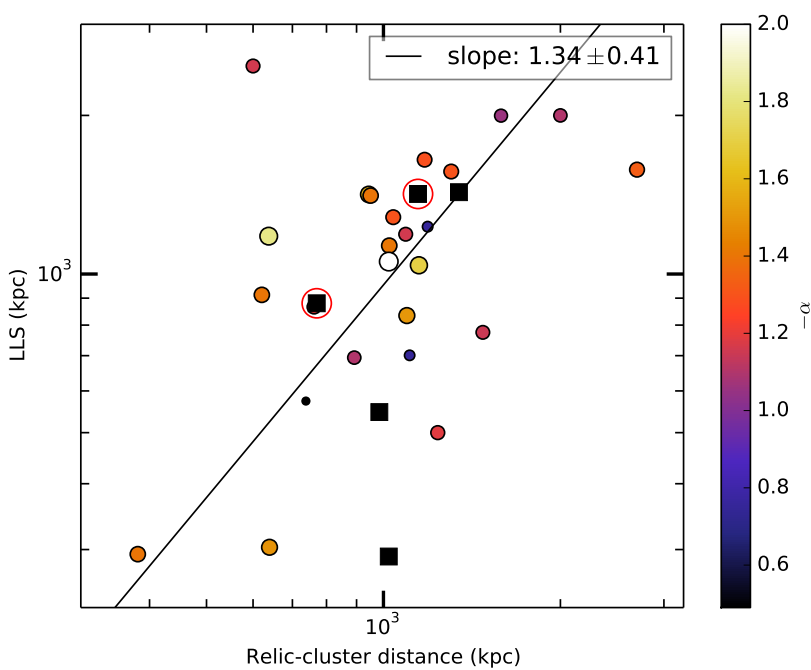

(a)

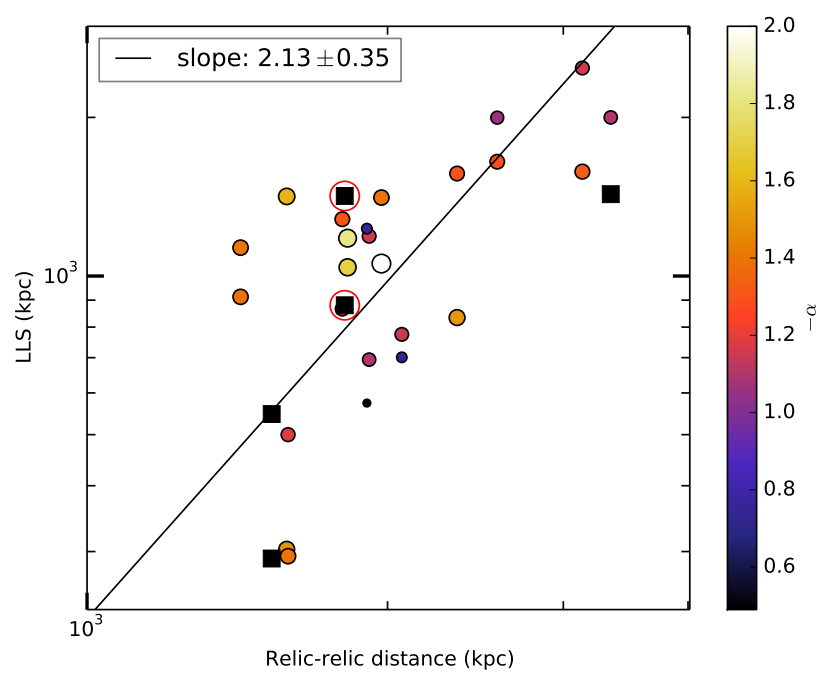

(b)

Figure 6. Left panel: LLS versus the projected distance between the radio relics in double systems and the X-ray peak of the hosting cluster. Color-coded is the spectral index value, black squares are for unknown values of the spectral index. The solid line shows the linear regression. Red circles show the values for PSZ1 G096.89+24.17. Right panel: same as the left panel but the independent variable is now the projected distance between the two relics of each system.

caused by an observation bias. However, higher sensitivity surveys, as those planned with LOFAR, are required to address this issue satisfactorily.

\subsection{Clues from simulations}

If we assume that radio relics are caused by merger shocks and that the luminosity of radio relics is proportional to the total energy released in a cluster merger, we can attempt to find a theoretical justification for the observed relation.

Simulations (e.g., Poole et al. 2006) suggest that a fixed fraction of the total energy dissipated in a merger goes into the primary merger shocks. The energy released in a merger between clusters of mass $M$ is given by $E \propto M^{2} / R_{\text {vir }}$, where $R_{\text {vir }}$ is the virial radius that is proportional to $R_{\mathrm{vir}} \propto M^{1 / 3}$. If the observed radio power scales as the dissipated kinetic energy flux, then the luminosity of the relics is $P \propto E / t_{\text {cross }}$, where $t_{\text {cross }}$ is the sound crossing time of the cluster (this is an assumption for the lifetime of the relics). The sound crossing time is $R_{\mathrm{vir}} / c_{\mathrm{s}}$, where $c_{\mathrm{S}}$ is the speed of sound which is $\propto T^{1 / 2}$. According to the mass-temperature relation (e.g., Pratt et al. 2009), $T \propto M^{2 / 3}$ so that $t_{\text {cross }} \propto M^{0}=$ const. Hence, this simple argument would predict a relation of the kind $P \propto M^{5 / 3}$. This is shallower than the slope of 2.8 found above. The discrepancy could be caused by the dependence of the particle acceleration efficiencies or the magnetic fields at the relics on some variable related to the cluster mass. Alternatively, this could be the result of observational bias, or the life-time of the relic depends on the cluster mass. Possibly, more insights may be gained from simulations.

The relation between the power of radio relics and the host cluster mass and luminosity has been investigated with cosmological simulations in the past (e.g. Hoeft et al. 2008; Pfrommer 2008 Skillman et al. 2011). However, previous work has been based on very small samples, often has made no clear distinction between radio relics and radio halos, and has been limited by numerical resolution.

Here we compare to a sample of clusters simulated with the AMR hydrodynamics code ENZO (Bryan et al. 2014) by Vazza et al. (2010), which consists of 20 galaxy clusters with masses in the range of $6 \times 10^{14} \leq M / M_{\odot} \leq 3 \times 10^{15}$ extracted from a total volume of $L_{\text {box }} \approx 480 \mathrm{Mpc} / \mathrm{h}$, and probing down to a resolution of $\approx 25 \mathrm{kpc} / \mathrm{h}$ for most of the virial volume. We analysed this cluster sample at redshifts $z=0, z=0.3$ and $z=0.6$, and extracted the Mach number and kinetic energy flux across shocks measured in the 3D distribution of gas (as in Vazza et al. 2012). Then we assigned a radio power to each shocked cell based on the model of Hoeft \& Bruggen (2007). To do so, we explored different models for the distribution of magnetic fields: a) uniform fields with $B=1 \mu \mathrm{G}$ everywhere (Buni model); b) the magnetic field model derived by Bonafede et al. (2010) as a best fit for the magnetic field profile of the Coma cluster (Bderiv model); c) we assigned to each shocked cell in the simulation a magnetic field corresponding to a $10 \%$ equipartition with the thermal gas energy, $B_{\text {eq }}=\sqrt{0.1 E_{g} \cdot 8 \pi}$, where $E_{g}$ is the thermal gas energy of each cell (Bequip model). This way, the (maximum) local amplification of the magnetic field is related to the thermal energy density of the ICM and to the host cluster mass. For each object and redshift we generated mock radio observations, and analysed them with a 2D relic detection scheme, which identifies as part of the same relic all pixels brighter than a given threshold as in Vazza et al. (2012). This way we can select objects with a clear presence of large double relic systems and compare them to the sample of observed relics.

Fig. 9 shows the comparison between the total radio power of the observed systems together with the best-fit of simulated relics for each model. Using the total radio emission instead of the power of the two radio relics separately is more robust against possible numerical uncertainties related to the specific criterion used to excise the radio power of each relic object in the simulated image. Furthermore, it is motivated by the scenario in which the total energy dissipated by each couple of relics follow the same merger event. While the normalization of the relation is dependent on the values of the magnetic field and of the electron acceleration efficiency (e.g., Hoeft \& Bruggen 2007), the slope is not. Therefore, we renormalized all simulated best-fit relations in Fig. 9 to match the observations best-fit at $M_{500}=5 \times 10^{14} M_{\odot}$. The figure shows that while the fixed magnetic field model is compatible with the 


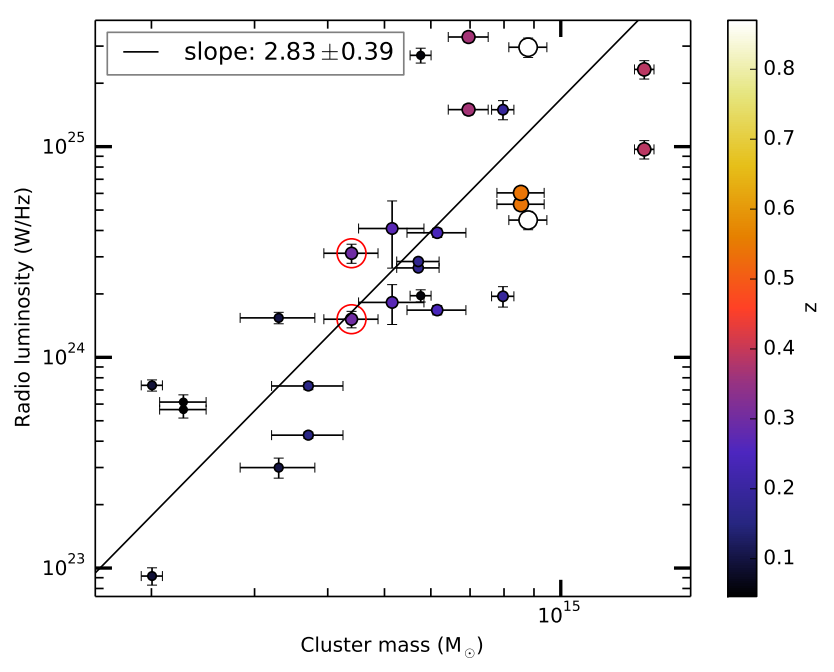

(a) Double radio relic systems

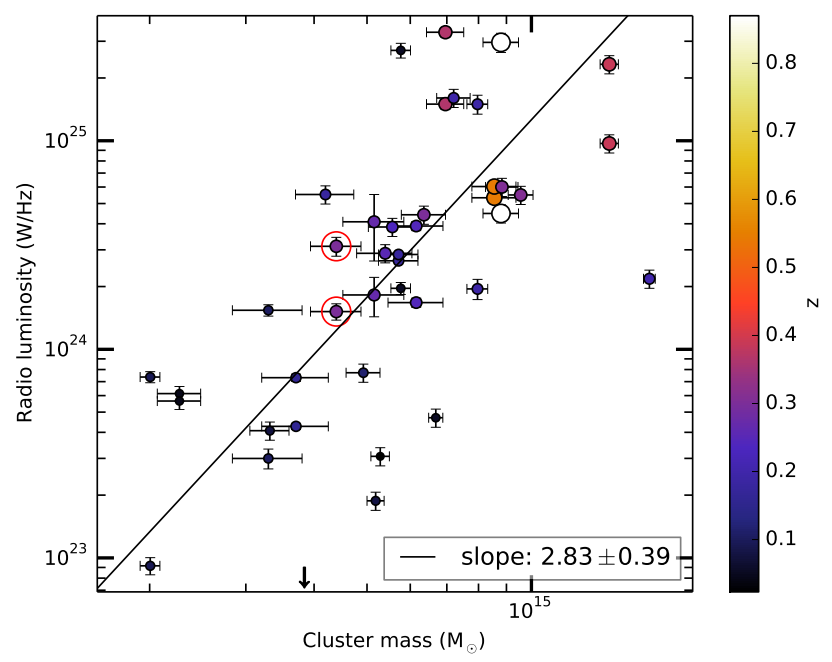

(b) Double + single radio relic systems

Figure 7. Cluster mass against radio relic power. Color coded the cluster redshift. Solid line is the linear regression. Red circles show the values for PSZ1 G096.89+24.17. Top: for double radio relics presented in table 1 Bottom: for double radio relics plus all relics of Feretti et al. (2012) marked as "extended" for which the mass is available from Planck SZ measurements. The arrow indicates the peculiar case of Abell 2146 where a clear shock is detected in the X-ray without a radio counterpart (Russell et al.|2011).

observed scaling $\left(L_{1.4 \mathrm{GHz}} \propto M^{3.0 \pm 1.0}\right)$, the other two models yield steeper relations $(4.73 \pm 1.74$ for the Bderiv model and $6.43 \pm 1.84$ for the Bequip model), incompatible with the observed relation. So it may be that the magnetic field does not vary much across the clusters in the sample and a strength of $\sim 2 \mu \mathrm{G}$ provides a reasonable fit to the observed relation.

The simulated $\left(L_{\mathrm{r}}, M\right)$ relation is steeper than the analytical estimate previously given $\left(L_{r} \propto M^{5 / 3}\right)$, even assuming a fixed magnetic field strength. The likely reason is that simulated radio relics always follow cluster-cluster mergers, where significant departures from the virial scaling previously presented are observed. In particular, simulated mergers present an abrupt increase of X-ray emission and temperature (e.g., Poole et al. 2006), due to the fast dissipation of kinetic energy in merger shocks. The same process should lead to an even sharper increase of the radio power in relics (Skillman et al. 2013), whose dependence on cluster mass is more com-

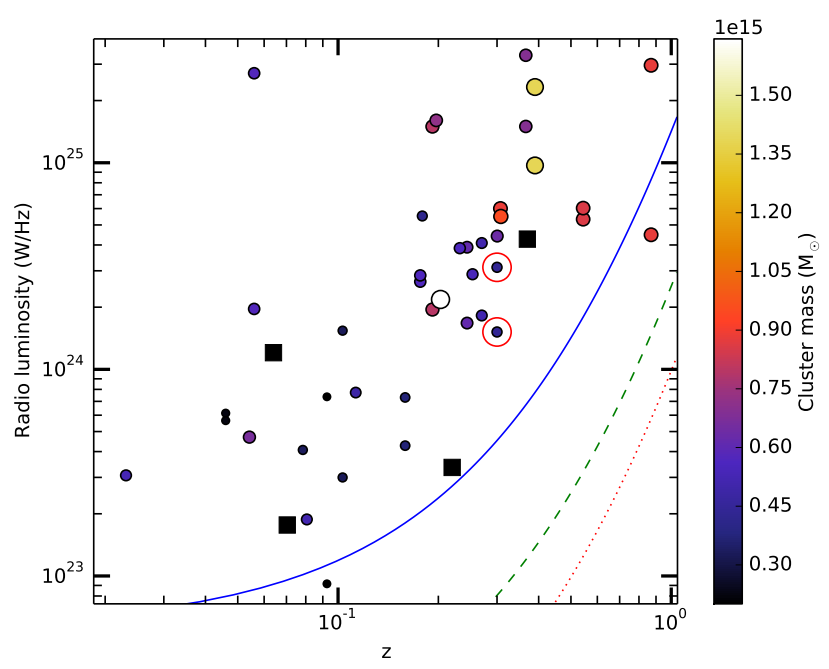

Figure 8. Cluster redshift versus radio relic luminosity for all double radio relics listed in Table 1 and all single relics presented in Feretti et al. (2012) marked as "extended". Black squares are clusters for which the Planck SZ catalogue does not provide a mass estimate. Solid blue line is an estimated $1 \sigma$ detection limit for relic detection in NVSS, green dashed line is the confusion limit for the VLA in D-configuration (which can be reached in 1 minute observation with the EVLA), while red dotted line is for LOFAR Tier- 1 (rms $0.1 \mathrm{mJy}$ and resolution $\left.25^{\prime \prime} \times 25^{\prime \prime}\right)$. Red circles show the values for PSZ1 G096.89+24.17.

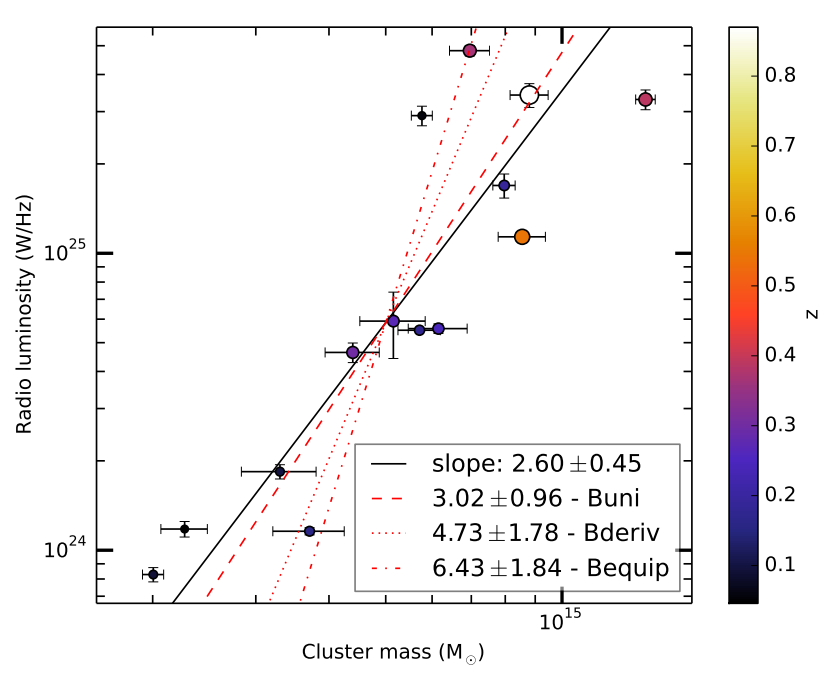

Figure 9. Same as in Fig. 7a but the power of radio relics belonging to the same clusters is added up. Red dashed line show the relation as predicted by simulations for a uniform magnetic field. Dotted line is using the magnetic field derived by the best fit of the Coma cluster data (Bonafede et al. 2010. Dash-dotted line is $10 \%$ of equipartition. Model lines were rescaled to match the data linear fit at $M=5 \times 10^{14} \mathrm{M}_{\odot}$

plex than derived from virial relations. However, we note that the normalisation is also changed by assuming a different acceleration efficiency for the electrons (which is of order of $\sim 10^{-4}$ here). As a cautionary remark, one should note that recent works of the radio and $\gamma$-ray properties of clusters that have double relics (Vazza \& Bruggen 2014) has cast doubts on the validity of the standard shock acceleration scenario that we just explored with this set of simulations. 


\section{CONCLUSIONS}

We have reported the discovery of a double radio relic system in the galaxy cluster PSZ1 G096.89+24.17. We present WSRT observations of the cluster at $1.4 \mathrm{GHz}$ which show two giant radio relics with a linear size of $0.9 \mathrm{Mpc}$ and $1.4 \mathrm{Mpc}$ and a hint of the presence of a radio halo. The short distance from the cluster centre and the rotated polarization angle for the southern relic suggest that the merger axis is slightly tilted compared to the plane of the sky, with the southern relic farther away from the observer.

We have compiled a comprehensive catalogue of all the known double radio relics in the literature. Compared to single radio relics, double systems are rarely misclassified radio phoenices and their projected distance are likely close to the real one. Using this catalogue we revisited the scaling relations investigated by van Weeren et al. (2009c) and Bonafede et al. (2012). Compared with previous works, we found no correlation between the LLS and the spectral index, but we confirm the correlation between the LLS and the relic distance from the cluster centre, in line with the idea that in the periphery of the clusters the shock surfaces are larger.

Our catalogue was then cross-matched with the cluster masses provided by the SZ measurements made by Planck. We found a good correlation between the radio relic luminosities and the cluster masses $\left(L_{r} \propto M^{2.83 \pm 0.39}\right)$. The relation could be reproduced in cosmological simulations and suggests that the magnetic field at the location of radio relics is rather uniform in all relics (within a factor of a few, which would explain the observed scatter around the mean), even though their masses, radial distances and Mach numbers are different.

\section{ACKNOWLEDGEMENTS}

The authors thank Gianfranco Brunetti for the useful discussions. $\mathrm{AB}$ and $\mathrm{MB}$ acknowledge support by the research group FOR 1254 funded by the Deutsche Forschungsgemeinschaft: "Magnetisation of interstellar and intergalactic media:the prospects of lowfrequency radio observations".

\section{REFERENCES}

Akritas M. G., Bershady M. A., 1996, The Astrophysical Journal, 470, 706

Bagchi J., Sirothia S. K., Werner N., Pandge M. B., Kantharia N. G., Ishwara-Chandra C. H., Paul S., Joshi S., 2011a, The Astrophysical Journal, 736, L8

Bagchi J. et al., 2011b, Memorie della Societa Astronomica Italiana, 82, 561

Blandford R., Eichler D., 1987, Physics Reports, 154, 1

Bonafede A. et al., 2012, Monthly Notices of the Royal Astronomical Society, 426, 40

Bonafede A., Feretti L., Murgia M., Govoni F., Giovannini G., Dallacasa D., Dolag K., Taylor G. B., 2010, Astronomy and Astrophysics, 513, A30

Bonafede A., Giovannini G., Feretti L., Govoni F., Murgia M., 2009, Astronomy and Astrophysics, 494, 429

Bonafede A., Intema H. T., Brüggen M., Girardi M., Nonino M., Kantharia N., van Weeren R. J., Röttgering H. J. A., 2014, Astrophysical Journal, 785

Boschin W., Barrena R., Girardi M., 2010, Astronomy and Astrophysics, 521, A78
Brown S., Duesterhoeft J., Rudnick L., 2011, The Astrophysical Journal, 727, L25

Brown S., Rudnick L., 2011, Monthly Notices of the Royal Astronomical Society, 412, 2

Bryan G. L. et al., 2014, The Astrophysical Journal Supplement Series, 211, 19

Cohen A. S., Lane W. M., Cotton W. D., Kassim N. E., Lazio T. J. W., Perley R. A., Condon J. J., Erickson W. C., 2007, The Astronomical Journal, 134, 1245

Condon J. J., Cotton W. D., Greisen E. W., Yin Q. F., Perley R. A., Taylor G. B., Broderick J. J., 1998, The Astronomical Journal, 115,1693

Drury L. O., 1983, Reports on Progress in Physics, 46, 973

Feretti L., Giovannini G., Govoni F., Murgia M., 2012, The Astronomy \& Astrophysics Review, 20, 54

Giovannini G., Bonafede A., Feretti L., Govoni F., Murgia M., 2010, Astronomy and Astrophysics, 511, L5

Giovannini G., Bonafede A., Feretti L., Govoni F., Murgia M., Ferrari F., Monti G., 2009, Astronomy and Astrophysics, 507, 1257

Hoeft M., Bruggen M., 2007, Monthly Notices of the Royal Astronomical Society, 375, 77

Hoeft M., Brüggen M., Yepes G., Gottlöber S., Schwope A., 2008, Monthly Notices of the Royal Astronomical Society, 391, 1511

Johnston-Hollitt M., 2004, Proceedings of The Riddle of Cooling Flows in Galaxies and Clusters of Galaxies

Jones F. C., Ellison D. C., 1991, Space Science Reviews, 58, 259

Kale R., Dwarakanath K. S., Bagchi J., Paul S., 2012, Monthly

Notices of the Royal Astronomical Society, 426, 1204

Lindner R. R. et al., 2013, eprint arXiv:1310.6786, 22

Menanteau F. et al., 2012, The Astrophysical Journal, 748, 7

Nuza S. E., Hoeft M., van Weeren R. J., Gottlöber S., Yepes G., 2012, Monthly Notices of the Royal Astronomical Society, 420, 2006

Offringa A. R., van de Gronde J. J., Roerdink J. B. T. M., 2012, Astronomy \& Astrophysics, 539, A95

Oppermann N. et al., 2012, Astronomy \& Astrophysics, 542, A93

Perley R. A., Butler B. J., 2012, arXiv:1211.1300, 56

Pfrommer C., 2008, Monthly Notices of the Royal Astronomical Society, 385, 1242

Planck Collaboration, Dole H., Gregorio A., Harrison D., Hempel A., 2013, Arxiv 1303.5089P, 42

Poole G. B., Fardal M. A., Babul A., McCarthy I. G., Quinn T., Wadsley J., 2006, Monthly Notices of the Royal Astronomical Society, 373, 881

Pratt G. W., Croston J. H., Arnaud M., Böhringer H., 2009, Astronomy and Astrophysics, 498, 361

Rau U., Cornwell T. J., 2011, Astronomy and Astrophysics, 532, A71

Rengelink R. B., Tang Y., de Bruyn A. G., Miley G. K., Bremer M. N., Rottgering H. J., Bremer M. A., 1997, Astronomy \& Astrophysics Supplement Series, 124, 259

Roettiger K., Burns J. O., Stone J. M., 1999, The Astrophysical Journal, 518, 603

Rottgering H. J. A., Wieringa M. H., Hunstead R. W., Ekers R. D., 1997, Monthly Notices of the Royal Astronomical Society, 290, 577

Russell H. R. et al., 2011, Monthly Notices of the Royal Astronomical Society: Letters, 417, L1

Skillman S. W., Hallman E. J., O'Shea B. W., Burns J. O., Smith B. D., Turk M. J., 2011, The Astrophysical Journal, 735, 96

Skillman S. W., O'Shea B. W., Hallman E. J., Burns J. O., Norman 
M. L., 2008, The Astrophysical Journal, 689, 1063

Skillman S. W., Xu H., Hallman E. J., O'Shea B. W., Burns J. O., Li H., Collins D. C., Norman M. L., 2013, The Astrophysical Journal, 765, 21

Stroe A., van Weeren R. J., Intema H. T., Röttgering H. J. A., Brüggen M., Hoeft M., J V. W. R., 2013, Astronomy \& Astrophysics, 555, A110

van Weeren R. J., Bonafede A., Ebeling H., Edge A. C., Brüggen M., Giovannini G., Hoeft M., Röttgering H. J. A., 2012a, Monthly Notices of the Royal Astronomical Society, 425, L36 van Weeren R. J., Brüggen M., Röttgering H. J. A., Hoeft M., 2012b, Journal of Astrophysics and Astronomy, 32, 505

van Weeren R. J., Brüggen M., Röttgering H. J. A., Hoeft M., Nuza S. E., Intema H. T., van Weeren R. J., 2011a, Astronomy \& Astrophysics, 533, A35

van Weeren R. J., Hoeft M., Röttgering H. J. A., Brüggen M., Intema H. T., van Velzen S., Weeren R. J. V., Velzen S. V., 2011b, Astronomy \& Astrophysics, 528, A38

van Weeren R. J. et al., 2009a, Astronomy and Astrophysics, 506, 1083

van Weeren R. J., Röttgering H. J. A., Brüggen M., Cohen A., 2009b, Astronomy and Astrophysics, 508, 75

van Weeren R. J., Röttgering H. J. A., Brüggen M., Hoeft M., 2010, Science, 330, 347

van Weeren R. J. V., Röttgering H. J. A., Brüggen M., Cohen A., van Weeren R. J., 2009c, Astronomy and Astrophysics, 508, 75

Vazza F., Bruggen M., 2014, Monthly Notices of the Royal Astronomical Society, 437, 2291

Vazza F., Brüggen M., van Weeren R., Bonafede A., Dolag K., Brunetti G., 2012, Monthly Notices of the Royal Astronomical Society, 421, 1868

Vazza F., Brunetti G., Gheller C., 2009, Monthly Notices of the Royal Astronomical Society, 395, 1333

Vazza F., Brunetti G., Gheller C., Brunino R., 2010, New Astronomy, 15,695

Venturi T., Giacintucci S., Brunetti G., Cassano R., Bardelli S., Dallacasa D., Setti G., 2007, Astronomy and Astrophysics, 463, 937

Vikhlinin a. et al., 2009, The Astrophysical Journal, 692, 1060

This paper has been typeset from a $\mathrm{T}_{\mathrm{E}} \mathrm{X} / \mathrm{LT}_{\mathrm{E}} \mathrm{X}$ file prepared by the author. 